\title{
Robust sEMG Electrodes Configuration for Pattern Recognition based Prosthesis Control
}

\author{
Yinfeng Fang and Honghai Liu \\ School of Computing \\ University of Portsmouth \\ Portsmouth, United Kingdom \\ yinfeng.fang@port.ac.uk, honghai.liu@port.ac.uk
}

\begin{abstract}
Electromyographic (EMG) signal is the electrical manifestation of a muscle contraction. Surface EMG signal can be obtained by electrodes on the skin to control prosthetic hand. However, surface EMG is sensitive to environmental interference, which leads to a low motion recognition rate of prosthesis control when encountering unexpected interferences, like electrodes shift. Electrodes shift occurs particularly in the day-to-day use of wearing electrodes. As a reslut, a long-term training procedure is necessary. To solve this problem, this paper proposes a new sEMG electrodes configuration to reduce the interference caused by electrodes shift. Experiments are designed to verify the improvements through evaluating the classification accuracy of discriminating eleven hand motions by pattern recognition approach. The comparison results show that the proposed electrodes configuration increases the pattern recognition rate by $4 \%$ and 8\% when applied kNN and LDA classifier, respectively. This paper suggests that optimising electrodes configuration is able to improve the EMG pattern discrimination and the proposed electrodes configuration has reference value.
\end{abstract}

Index Terms-Surface EMG, Electrodes Shift, Prosthesis, Hand Motion, Pattern Recognition

\section{INTRODUCTION}

A myoelectric signal, or electromyogram (EMG), is the electrical manifestation of a muscle contraction[1]. In detail, the EMG signal is the summation of the action potentials discharged by the active muscle fibers in the proximity of the recording electrodes[1]. One promising application of EMG is prosthetic extremes control, since EMG signals captured from the arm of patients are originally the control signals of the human hand. Therefore, EMG may supply a natural and intuitive approach to help amputees to control prosthetic hand. Researches on EMG based prosthetic extremes control can be found in [2], [3], [4]. In addition, a variety of myoelectric prosthetic hands have been appeared in the market recent years, like BeBionic3 (Steeper 1td., UK), i-limb ultra (Touch Bionics, Uk), and Michelangelo[5].

EMG signal is generally divided into two groups: surface EMG (sEMG) and needle EMG (also be known as intramuscular EMG or internal EMG). Needle EMG is of high selectivity, and is less representative of the global muscle activity and thus may provide precise control signal[6]. However, needle EMG is invasive, thus few researchers apply needle EMG signal for prosthetic hand control. Surface EMG, a technique by which muscle activation potentials are gathered by electrodes placed on the patients forearm skin; these potentials can be used to track which muscles the patient is willing to activate, and with what force[7]. In principle, sEMG is a cheap and easy way of detecting what the patient wants the prosthesis to do [7]. Although needle EMG have less muscular crosstalk allowing for more independent control sites, the results show that there is no significant difference in classification accuracy in wrist and grip movements[8].

EMG signal is non-stationary[9] and easy to be contaminated by a wide variety of factors, not only including equipments inherent noise, ambient noises and motion artefacts, but also physiological and anatomical properties[10], which would lead to the changes in EMG patterns for prosthetic control. Therefore, advanced signal processing technology should be considered to deal with the variability of EMG signal. Interpreted by [8], there are two major approaches that can be pursued to increase the accuracy of prosthetic controllers: 1) use signal processing to extract more information from the input signals; or 2) provide more informative raw signals to the controller.

The placement of sEMG electrodes is a critical issue for the successful identification of EMG patterns. Generally, the configuration of surface electrodes falls into three groups: 1) Traditional configuration, which requires to pinpoint muscles and adhere pairs of electrodes on the belly of them. The process of identification is highly inefficient and inconvenient because users typically have no knowledge about muscle distribution[11]. Besides, the recognition accuracy is highly depends on the precise electrodes placement. 2) Low-Density surface electrode layout[12], [13], which arranges electrodes evenly on the skin[14], [11]. When apply LD electrode configuration on the arm to identify hand motion, the electrodes usually form a ring or belt structure with more than two EMG channels. In this approach, user is regardless of the muscle anatomy. 3) Hight-Density surface electrodes layout is the enhanced version of the LD one with much higher electrodes density. The interval-electrode distance is designed less than $10 \mathrm{~mm}$ to avoid the influence on the EMG pattern caused by the inherent spatial filter. However, the coverage area on the skin is limited due to the small interval-distance. Besides, the application field of HD-sEMG is mostly in single motor units activities analysis [15], [16], [17], which is far from the topic of prosthetic hand control.

Low-Density surface electrode layout would be the trade- 
off to capture sEMG signal for myoelectric control, which eliminates the process of muscle identification and owns less electrodes and cover more area than HD-EMG. Atzori , etc.,[18] show that, to extract discriminable patterns from EMG signal, precise placement of electrodes on specific muscles (traditional electrodes layout) is not required. Castellini , etc., [19] demonstrated that the same uniform electrode positioning strategy (Low-density electrodes layout) works fine for all tested subjects, notwithstanding their diversity. Several papers [13], [20], [21], [22], [23] make the same conclusion that the classification accuracies improve with an increased number of electrodes to a certain point at which increases in classification accuracy start to diminish with more electrodes. But the specific turn point of the electrodes number varies with different electrode layout. In healthy subjects, reducing the number of electrodes to eight did not affect accuracy significantly when those electrodes were optimally placed, but did reduce accuracy significantly when those electrodes were distributed evenly[9], [22]. In the amputee subjects, reducing the number of electrodes up to a certain point did not affect classification accuracy or the number of tasks with high accuracy, independent of whether those remaining electrodes were evenly distributed or optimally placed[22].

Recent years have witnessed the fast development of dexterous multiple degrees-of-freedom (DoF) and sensory equipped prosthetic hands by researchers and manufacturers [24], [25], [26]. As efforts in the development of prosthetic hands with high dexterity come to fruition, it will be necessary for the appropriate control systems to follow suit [27]. Although modern prosthetic hands such as I-Limb(Touchbionics, USA) have the provision of controlling individual fingers, the user interface is still hardly to control individual fingers[28]. To ensure a massive clinical evaluation and their commercial exploitation, the prosthetic hands should be capable of a robust, reliable, and intuitive control interface, allowing dexterous control [29], [30]. However, the communication interface between the user and the machine is the technological bottle-neck which explains why current hand prostheses are very simple from a biomechanics point of view, even if more sophisticated solutions would be possible[31]. For most commercial myoprosthetic hands, two pairs of EMG electrodes are usually adopted due to the practical factors, like lower cost and weight and space limitations[32], lower power consumption[20].

It is a trend to control prosthesis using pattern recognition technology, which has the potential to provide better, more reliable control signals. However, proper training is essential. Simon, etc., [33] classify the procedure of training into four stages: conceptual training stage, control training stage, functional use training stage and prosthesis recalibration training stage, of which prosthesis recalibration training involves teaching the individual how to retain functional use of their pattern recognitioncontrolled prosthesis if control degrades during daily use. Differences in prosthesis donning, environmental changes, muscle fatigue, and electrode problems can all occur during day-to-day use. Prompting the individual to move the prosthesis throughout its full range of motion after donning

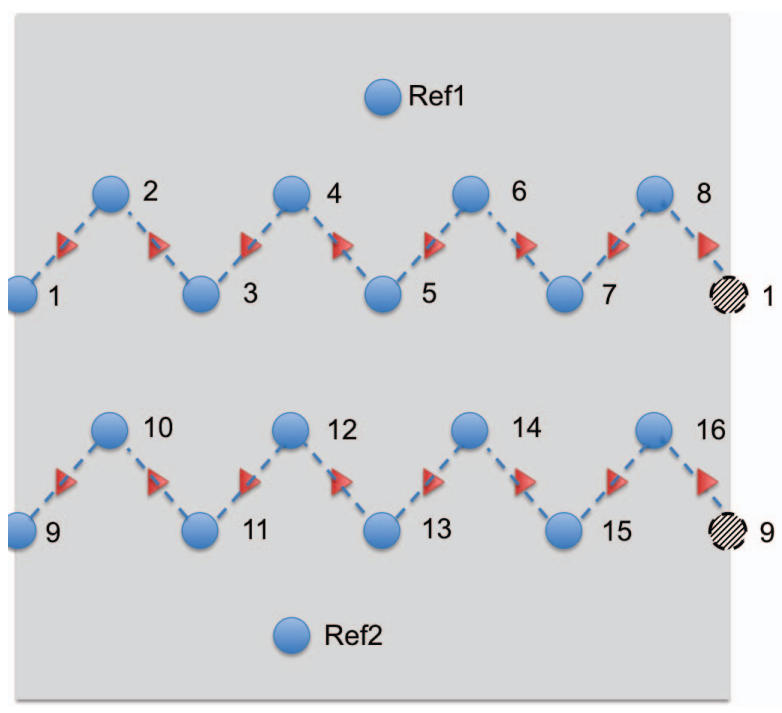

Fig. 1. This figure shows the expanded view of electrodes configuration that proposed in this paper, named zig Configuration. Blue solid circules labelled from 1 to 16 stand for sEMG electrodes. The red triangle indicates a bipolar sEMG channel connecting two neighbour electrodes connected by dot lines. Electrode Ref1 and Ref2 are the reference electrodes. The dark background indicate the elastic fabric where all the electrodes are fixed on it in advance.

will allow him or her to test their control and determine if recalibration is necessary[33]. The long-time training procedure has become one of the main reasons leading to high rejection rate of wearing prosthetic hand[34].

To reduce the training time, several approaches can be attempted: 1) developing robust sEMG features that can satisfy long-term use [10], 2) implement on-line training and recognition methods[35], [36] and 3) development of robust sEMG acquisition system that is able to compensate the fault caused by different prosthesis donning. It is the third way that this paper considers. In the premise of using the same number of electrodes, this paper proposes a new electrodes configuration than can enhance the robustness of system, especially when existing electrode shift.

\section{MAteriAl AND METHODS}

\section{A. Apparatus}

To measure the surface EMG signal on the forearm, a 16 channel sEMG acquisition system is employed[37]. Based on the acquisition system, two types of electrodes configuration are designed in this paper, as seen in Fig.1 and Fig.2. Zig configuration is the new one proposed in this paper, and parallel configurations are widely applied in LD electrodes configuration.

Comparing with traditional surface EMG technology that needs to stick pairs of electrodes on interested muscles, both configuration of electrodes used in this paper ignore the muscle positions. In healthy people, 11 muscles control motionos of each finger and the wrist in anatomy. They are Extensor Carpi Uinaris (ECU), Extensor Digitorum (ED), Extensor Carpi Radiails Brevis(ECRB), Extensor Digiti Minimi (EDM), Flexor Carpi Ulnaris (FCU), Flexor Digitorum Profundus 


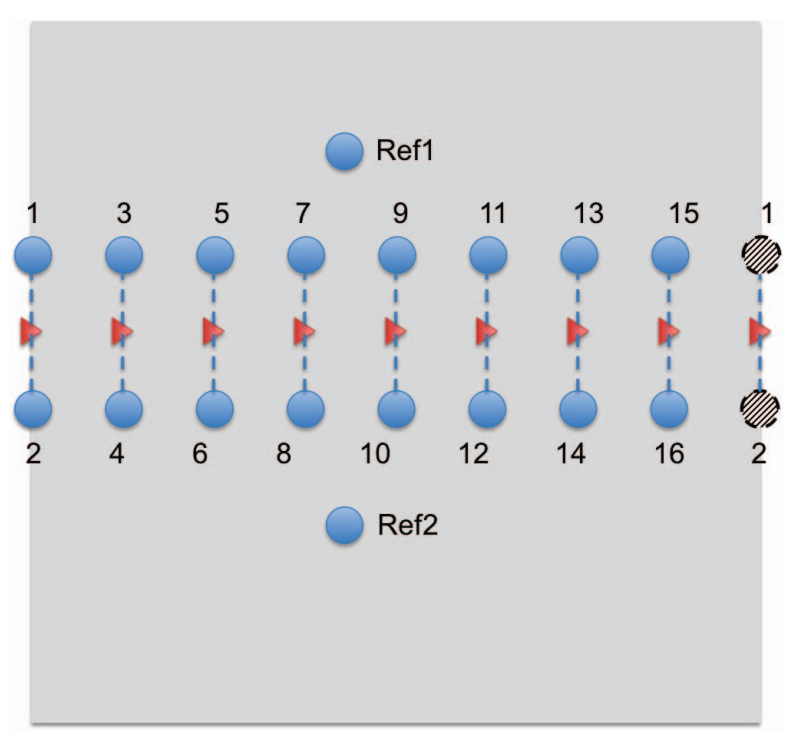

Fig. 2. This figure shows the expanded view of electrodes configuration in traditional sEMG configuration, named parallel configuration. Blue solid circles labelled from 1 to 16 stand for sEMG electrodes. The red triangle indicates a bipolar sEMG channel connecting two neighbour electrodes connected by dot lines. Electrode Ref 1 and Ref 2 are the reference electrodes. The dark background indicate the elastic fabric where all the electrodes are fixed on it in advance.

(FDP), Abductor Pollicis Longus (APL), Extensor Pollicis Brevis (EPB), Extensor Pollicis Longus (EPL) and Extensor Indicis (EE). The distribution of these muscles are complex. Some muscles are near to the skin, thus the surface EMG signals from them are stronger than those the signals stemming from the muscles inside of the forearm. Therefore, it is not possible to pinpoint all the muscle and put corresponding EMG electrodes on them. Furthermore, crosstalk is a considerable issue in traditional sEMG technology as reported in [38]. To resolve these problems, a more general strategy is proposed by using a specific electrodes configuration, which takes all muscles as the sources for each EMG channel, seen in Equation 1.

$$
E_{j}=\sum_{i=1}^{11} a_{i j} S_{i} \quad j=1 \cdots 16,
$$

where $E_{j}$ is the EMG signal measured from channel $j, S_{i}$ indicates the clean EMG signal of the $i^{\text {th }}$ muscle and $a_{i j}$ are the coefficients that demonstrate its weight to $E_{j}$ from muscle $i$. Fig. 3 shows that the multi-channel EMG signal stems from multi-muscle sources.

In this paper, the EMG signal amplifier factor is 5000 and the signal is filtered by a band pass filter from $20 \mathrm{~Hz}$ to 500 $\mathrm{Hz}$, and meanwhile $50 \mathrm{~Hz}$ power-line noise is suppressed by a notch filter in hardware. The sampling frequency is $1 \mathrm{kHz}$ and the ADC resolution is 12 bits. The EMG device is connected to a PC that installed Windows XP operating system via USB port, and a custom designed software is used to save the sEMG data. To remove the power-line noise and its harmonic component further, a comb filter is enabled in the software.

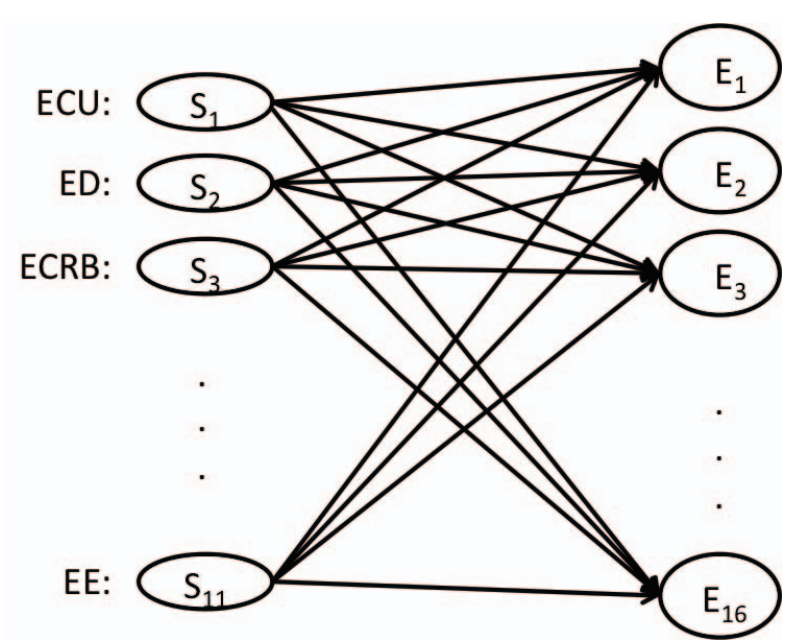

Fig. 3. This figure demonstrates that the muscle sources of EMG signals measured by 16 channels.

\section{B. Subjects}

Two right-handed university staff served as subjects. [subject 1, age: 41 , mass: $72 \mathrm{~kg}$, height: $174 \mathrm{~cm}$ ][subject 2, age: 28 , mass: $62 \mathrm{~kg}$, height: $170 \mathrm{~cm}$ ]. The subjects had no previous history of neuropathies or traumas to the upper limbs. This project is approved by the ethic institution of University of Portsmouth.

\section{Data Collection}

Ten hand motions are designed for sEMG data collection, as seen in Fig.4. Six groups of motions were collected for each subject. Three groups (Group 1, Group 3, Group 5) were collected by the Zig configuration, seen in 1, and the other three groups (Group 2, Group 4, Group 6) were collected by parallel configuration, seen in Fig.2. For each group, ten hand motions (motion 1 to motion 10) were performed and each motion repeat 10 times according to the given cue signal. A repeat also calls a trial. Cue signals for motion starting is at $5 \mathrm{~s}, 15 \mathrm{~s}, 25 \mathrm{~s}, \ldots 95 \mathrm{~s}$, and Cue sign for motion stopping is at $10 \mathrm{~s}, 20 \mathrm{~s}, 30 \mathrm{~s}, \ldots, 100 \mathrm{~s}$. Fig. 5 shows one EMG signal channel in channel one, motion one, group three, subject two.

No pretreatment of the forearm skin is required before the subjects wear the electrodes sleeve, and no labels is required on the skin to avoid electrodes shift as did in [9]. Subjects are required to make sure that the reference electrodes stays on the upside of the forearm when keeping arm and palm upwards. After wearing the sleeve with electrodes, another empty sleeve with no electrodes is used to cover the previous sleeve for the sake of generating a squeeze to every electrodes towards the skin. It is suggested to wait for about 10 minutes in order to reduce the skin to electrodes resistance naturally. During data collection, the subjects were seated in a chair beside the experiment desk facing the computer monitor with the elbow on the armband, seen in Fig. 6. 


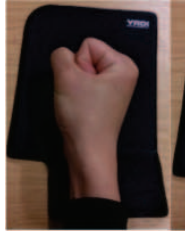

(1)

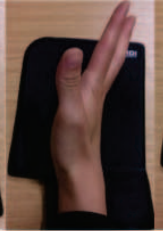

(2)

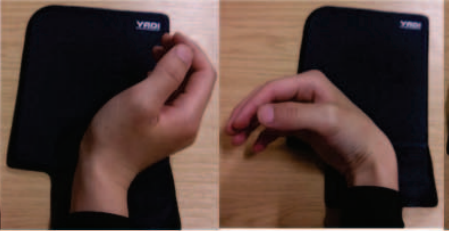

(3)
(4)

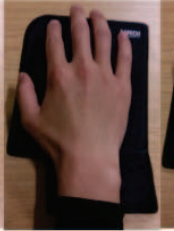

(5)

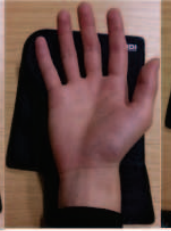

(6)

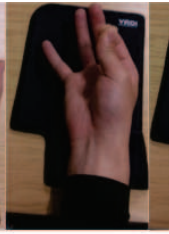

(7)

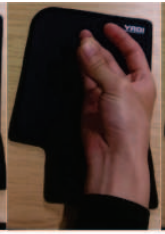

(8)

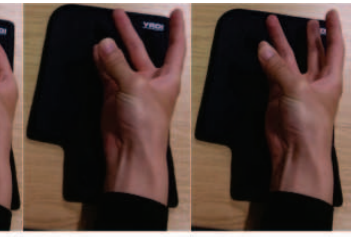

(9)

(10)

Fig. 4. This figure shows ten hand motions that are used in this paper for hand motion recognition. 1 Hand-Close, 2 Hand-Open, 3 Wrist-Flexion, 4 WristExtension, 5 Supination, 6 Pronation, and 7-10 using thumb to touch index finger, middle finger, ring finger and little finger.

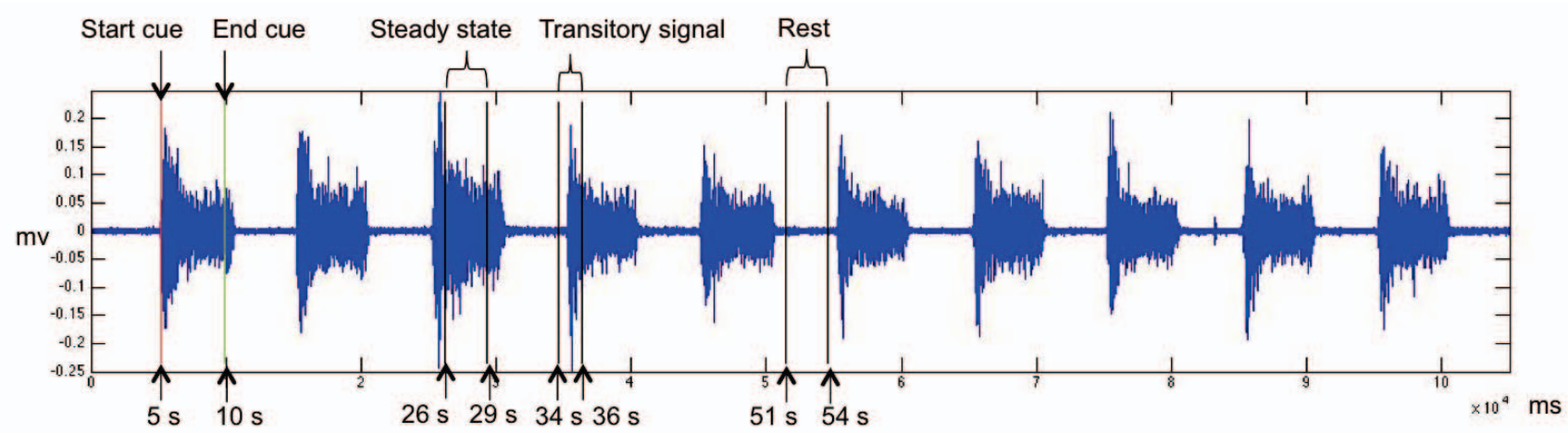

Fig. 5. This picture shows the EMG signal in channel one, motion one, group three captured from subject 2. The Start cue and End cue can be found in this figure and the steady-state, transitory and rest state EMG signal are identified as well.

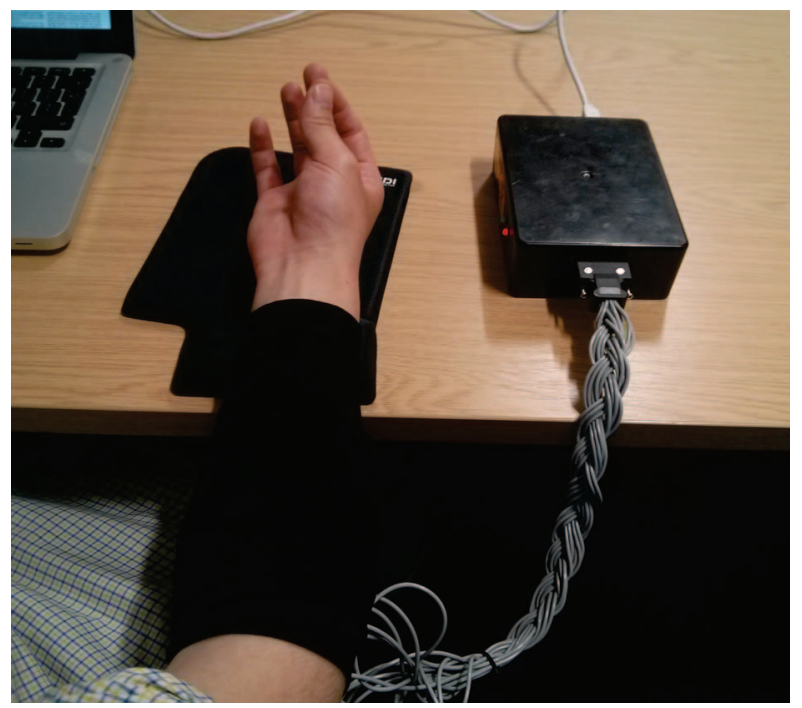

Fig. 6. It shows the scene of data collection from one subject.

\section{Signal processing}

The sEMG signals were segmented with a $300 \mathrm{~ms}$ window and $100 \mathrm{~ms}$ window shift for feature extraction. A $100 \mathrm{~ms}$ window shift is sufficient for real-time requirement. In this paper, LgSVM[37] and WL[9] were selected as sEMG feature. For each motion with 10 trials, only the steady-state signals were selected for classification. The transitory signals are not considered in this paper, because a prosthesis would not be able to respond to transitory signal due to mechanical inertia[39]. The start and end point of steady-state signals are 1 second after the cue signal of starting a hand motion and 1 second before the cue signal of the end signal of a hand motion. Some examples of these regions are [6s, 9s], [16s, 19s], etc. The sEMG signals of rest was also extracted in the regions of [11s, $14 \mathrm{~s}],[21 \mathrm{~s}, 24 \mathrm{~s}]$ and so forth. Therefore, the sample size of hand motions in one group is $30 \times 10 \times 10(30$ sample in one trial, 10 trials for one motion, 10 motions in total) $=3000$. For Group 1, Group 3 and Group 5, the dimension of one sample is 16 (16 channels) and for Group 2, Group 4 and Group 6, the dimension of one sample is 8. Because the dataset of rest signals are larger than other hand motions, this paper compresses the sample size of rest equaling to that of one hand motion through randomly selecting 300 samples. Thus, there are total 3300 samples with 11 groups (rest included) for classification for each group. The samples were fed to kNN and LDA for classification. Using one or two groups for training, and the rest groups for testing, termed as Cross-Group testing. The training sample and testing training are from the same electrodes configuration.

\section{RESULTS AND DISCUSSION}

The average Inner-group testing results for each group of both subjects are higher than $99 \%$, seen in table I. The average classification accuracy of using the proposed electrodes configuration is $99.65 \%$, and it is $99.49 \%$ for the traditional electrodes configuration. Thus, the recognition accuracies are very similar for both the electrodes configuration. However, 


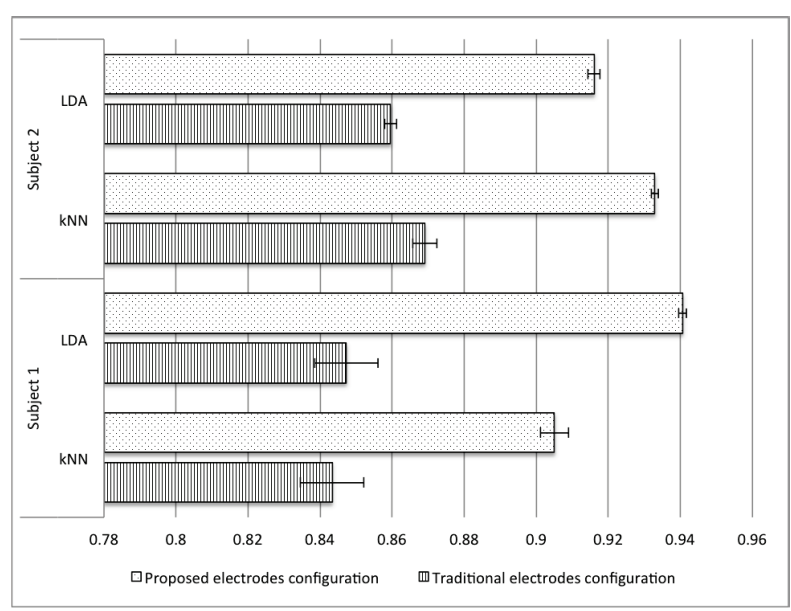

Fig. 7. Cross-group testing results

the $\mathrm{kNN}$ classifier outperforms LDA classifier in the average accuracies on discriminating hand motions in both subjects. It may conclude that classifiers play a more important role in hand motion recognition than electrodes configuration.

The Cross-Group testing results for subject 1 using the proposed electrodes configuration and the traditional configuration are 0.91/0.94 (kNN/LDA) and 0.87/0.84 (kNN/LDA), respectively. The same results for subject 2 are 0.93/0.92 (kNN/LDA) and $0.89 / 0.89$ (kNN/LDA), seen in Table II. There exists $4 \%$ $(\mathrm{kNN})$ and $8 \%$ (LDA) improvements when using the proposed electrodes configuration comparing with the traditional one in subject one. The same figures in subject 2 are 4\% and 3\%. The results clearly show that the proposed electrodes configuration achieves better classification performance than the traditional one in Cross-Group testing. There are no much difference in the use of different classifiers. In addition, the variance of the classification accuracy using the proposed electrodes configuration is lower than that of using traditional electrodes configuration, as seen in the bar chart Fig. 7, which also shows that the proposed configuration is more robust.

\section{CONCLUSION}

In the application of sEMG based dexterous prosthesis manipulation using pattern recognition approaches, electrodes shift deteriorate the recognition accuracy. To obtain robust sEMG signal for prosthesis control, this paper pays attention to electrodes configuration and proposed a novel way to organise 18 electrodes to form 16 sEMG channel, Zig electrodes configuration. It was compared with the parallel electrodes configuration using the same numbers of electrodes. The experiment results show that the proposed electrodes configuration improved the hand motion recognition accuracy by $4 \%$ when using $\mathrm{kNN}$ and by $8 \%$ when using LDA. Therefore, it suggests that optimising electrodes configuration is able to improve the EMG pattern discrimination, and the proposed electrodes configuration has reference value.

\section{REFERENCES}

[1] N. Jiang, D. Falla, A. d'Avella, B. Graimann, and D. Farina, "Myoelectric control in neurorehabilitation," Crit Rev Biomed Eng, vol. 38, no. 4, pp. 381-91, 2010, jiang, Ning Falla, Deborah d'Avella, Andrea Graimann, Bernhard Farina, Dario Crit Rev Biomed Eng. 2010;38(4):381-91.

[2] M. Zecca, S. Micera, M. C. Carrozza, and P. Dario, "Control of multifunctional prosthetic hands by processing the electromyographic signal," Crit Rev Biomed Eng, vol. 30, no. 4-6, pp. 459-85, 2002.

[3] M. Zardoshti-Kermani, B. C. Wheeler, K. Badie, and R. M. Hashemi, "Emg feature evaluation for movement control of upper extremity prostheses," Rehabilitation Engineering, IEEE Transactions on, vol. 3, no. 4, pp. 324-333, 1995.

[4] L. Hargrove, K. Englehart, and B. Hudgins, "A training strategy to reduce classification degradation due to electrode displacements in pattern recognition based myoelectric control," Biomed Signal Process Control, vol. 3, no. 2, pp. 175-180, 2008.

[5] ottobock, "http://www.living-with-michelangelo.com/gb/home/."

[6] E. N. Kamavuako, J. C. Rosenvang, M. F. Bg, A. Smidstrup, E. Erkocevic, M. J. Niemeier, W. Jensen, and D. Farina, "Influence of the feature space on the estimation of hand grasping force from intramuscular emg," Biomedical Signal Processing and Control, vol. 8, no. 1, pp. 1-5, 2013.

[7] C. Castellini and P. van der Smagt, "Surface emg in advanced hand prosthetics," Biol Cybern, vol. 100, no. 1, pp. 35-47, 2009.

[8] L. J. Hargrove, K. Englehart, and B. Hudgins, "A comparison of surface and intramuscular myoelectric signal classification," Ieee Transactions on Biomedical Engineering, vol. 54, no. 5, pp. 847-853, 2007.

[9] J. Rafiee, M. A. Rafiee, F. Yavari, and M. P. Schoen, "Feature extraction of forearm emg signals for prosthetics," Expert Systems with Applications, vol. 38, no. 4, pp. 4058-4067, 2011.

[10] A. Phinyomark, F. Quaine, S. Charbonnier, C. Serviere, F. TarpinBernard, and Y. Laurillau, "Emg feature evaluation for improving myoelectric pattern recognition robustness," Expert Systems with Applications, vol. 40, no. 12, pp. 4832-4840, 2013.

[11] X. Tang, Y. Liu, C. Lv, and D. Sun, "Hand motion classification using a multi-channel surface electromyography sensor," Sensors (Basel), vol. 12 , no. 2 , pp. 1130-47, 2012.

[12] S. Sakthibalan, R. Twycross-lewis, R. Woledge, Y. Hao, and D. Morrissey, "Low-density, high surface area electromyography of the hamstring muscles during running and kicking," British Journal of Sports Medicine, vol. 45, no. 2, 2011.

[13] G. Huang, Z. Zhang, D. Zhang, and X. Zhu, "Spatio-spectral filters for low-density surface electromyographic signal classification," Med Biol Eng Comput, vol. 51, no. 5, pp. 547-55, 2013.

[14] Y. C. Du, C. H. Lin, L. Y. Shyu, and T. S. Chen, "Portable hand motion classifier for multi-channel surface electromyography recognition using grey relational analysis," Expert Systems with Applications, vol. 37, no. 6, pp. 4283-4291, 2010.

[15] J. H. Blok, J. P. van Dijk, G. Drost, M. J. Zwarts, and D. F. Stegeman, "A high-density multichannel surface electromyography system for the characterization of single motor units," Review of Scientific Instruments, vol. 73, no. 4, pp. 1887-1897, 2002.

[16] R. Merletti, A. Holobar, and D. Farina, "Analysis of motor units with high-density surface electromyography," J Electromyogr Kinesiol, vol. 18, no. 6, pp. 879-90, 2008.

[17] E. M. Maathuis, J. Drenthen, J. P. van Dijk, G. H. Visser, and J. H. Blok, "Motor unit tracking with high-density surface emg," J Electromyogr Kinesiol, vol. 18, no. 6, pp. 920-30, 2008.

[18] M. Atzori, A. Gijsberts, S. Heynen, A. G. Hager, C. Castellimi, B. Caputo, and H. Muller, "Experiences in the creation of an electromyography database to help hand amputated persons," Stud Health Technol Inform, vol. 180 , pp. 828-32, 2012.

[19] C. Castellini, E. Gruppioni, A. Davalli, and G. Sandini, "Fine detection of grasp force and posture by amputees via surface electromyography," J Physiol Paris, vol. 103, no. 3-5, pp. 255-62, 2009.

[20] S. Muceli and D. Farina, "Simultaneous and proportional estimation of hand kinematics from emg during mirrored movements at multiple degrees-of-freedom,” IEEE Trans Neural Syst Rehabil Eng, vol. 20, no. 3, pp. 371-8, 2012.

[21] P. Zhou, M. M. Lowery, K. B. Englehart, H. Huang, G. Li, L. Hargrove, J. P. Dewald, and T. A. Kuiken, "Decoding a new neural machine interface for control of artificial limbs," J Neurophysiol, vol. 98, no. 5, pp. 2974-82, 2007. 
TABLE I

INNER-GROUP TESTING RESULTS

\begin{tabular}{c|c|ccc|ccc|c}
\hline & & Group1 & Group3 & Group5 & Group2 & Group4 & Group6 & AVG \\
\hline \multirow{2}{*}{ kNN } & subject 1 & $100.00 \%$ & $100.00 \%$ & $100.00 \%$ & $100.00 \%$ & $100.00 \%$ & $100.00 \%$ & $100.00 \%$ \\
& subject 2 & $99.97 \%$ & $100.00 \%$ & $100.00 \%$ & $100.00 \%$ & $99.97 \%$ & $100.00 \%$ & $99.99 \%$ \\
\multirow{2}{*}{ LDA } & subject 1 & $99.00 \%$ & $99.21 \%$ & $99.38 \%$ & $99.44 \%$ & $98.80 \%$ & $98.74 \%$ & $99.10 \%$ \\
& subject 2 & $99.27 \%$ & $99.03 \%$ & $99.91 \%$ & $99.33 \%$ & $98.38 \%$ & $99.27 \%$ & $99.20 \%$ \\
& AVG & & $99.65 \%$ & & & $99.49 \%$ & & $99.57 \%$ \\
\hline
\end{tabular}

TABLE II

Cross-Group testing RESUlts

\begin{tabular}{|c|c|c|c|c|c|c|c|c|c|c|c|c|}
\hline & Configuration & Classifiers & $\mathrm{g} 1 \rightarrow \mathrm{g} 2$ & $\mathrm{~g} 1 \rightarrow \mathrm{g} 3$ & $\mathrm{~g} 2 \rightarrow \mathrm{g} 1$ & $\mathrm{~g} 2 \rightarrow \mathrm{g} 3$ & $\mathrm{~g} 3 \rightarrow \mathrm{g} 1$ & $\mathrm{~g} 3 \rightarrow \mathrm{g} 2$ & $\mathrm{~g} 1+\mathrm{g} 2 \rightarrow \mathrm{g} 3$ & $\mathrm{~g} 1+\mathrm{g} 3 \rightarrow \mathrm{g} 2$ & $\mathrm{~g} 2+\mathrm{g} 3 \rightarrow \mathrm{g} 1$ & AVE \\
\hline \multirow{4}{*}{ Subject 1} & \multirow{2}{*}{ Zig } & $\mathrm{kNN}$ & 0.878 & 0.8138 & 0.8619 & 0.9543 & 0.8323 & 0.9727 & 0.9651 & 0.9713 & 0.8959 & 0.91 \\
\hline & & LDA & 0.9786 & 0.9545 & 0.8804 & 0.9015 & 0.9733 & 0.9346 & 0.939 & 0.9531 & 0.9507 & 0.94 \\
\hline & \multirow{2}{*}{ Parallel } & $\mathrm{kNN}$ & 0.6915 & 0.737 & 0.9211 & 0.9499 & 0.9384 & 0.907 & 0.9358 & 0.8337 & 0.8974 & 0.87 \\
\hline & & LDA & 0.8906 & 0.8768 & 0.8674 & 0.7452 & 0.9065 & 0.6311 & 0.9032 & 0.815 & 0.9023 & 0.84 \\
\hline \multirow{4}{*}{ Subject 2} & \multirow{2}{*}{$\mathrm{Zig}$} & $\mathrm{kNN}$ & 0.9238 & 0.9628 & 0.9572 & 0.9463 & 0.9273 & 0.8625 & 0.9355 & 0.9238 & 0.9572 & 0.93 \\
\hline & & LDA & 0.9334 & 0.9798 & 0.8968 & 0.9155 & 0.9188 & 0.8302 & 0.9399 & 0.9334 & 0.8968 & 0.92 \\
\hline & \multirow{2}{*}{ Parallel } & $\mathrm{kNN}$ & 0.8484 & 0.9633 & 0.8408 & 0.812 & 0.9299 & 0.8938 & 0.9065 & 0.8686 & 0.988 & 0.89 \\
\hline & & LDA & 0.8531 & 0.9346 & 0.8918 & 0.8554 & 0.9246 & 0.8589 & 0.8944 & 0.854 & 0.9622 & 0.89 \\
\hline
\end{tabular}

[22] H. Daley, K. Englehart, L. Hargrove, and U. Kuruganti, "High density electromyography data of normally limbed and transradial amputee subjects for multifunction prosthetic control," J Electromyogr Kinesiol, vol. 22, no. 3, pp. 478-84, 2012.

[23] A. Andrews, E. Morin, and L. McLean, "Optimal electrode configurations for finger movement classification using emg," Conf Proc IEEE Eng Med Biol Soc, vol. 2009, pp. 2987-90, 2009.

[24] C. Lake and R. Dodson, "Progressive upper limb prosthetics," Phys Med Rehabil Clin N Am, vol. 17, no. 1, pp. 49-72, 2006.

[25] S. A. Dalley, T. E. Wiste, T. J. Withrow, and M. Goldfarb, "Design of a multifunctional anthropomorphic prosthetic hand with extrinsic actuation," Ieee-Asme Transactions on Mechatronics, vol. 14, no. 6, pp. 699-706, 2009.

[26] D. A. Zlotolow and S. H. Kozin, "Advances in upper extremity prosthetics," Hand Clinics, vol. 28, no. 4, pp. 587-593, 2012.

[27] F. V. G. Tenore, A. Ramos, A. Fahmy, S. Acharya, R. EtienneCummings, and N. V. Thakor, "Decoding of individuated finger movements using surface electromyography," Ieee Transactions on Biomedical Engineering, vol. 56, no. 5, pp. 1427-1434, 2009.

[28] S. P. Arjunan and D. K. Kumar, "Decoding subtle forearm flexions using fractal features of surface electromyogram from single and multiple sensors," J Neuroeng Rehabil, vol. 7, p. 53, 2010.

[29] C. Cipriani, C. Antfolk, M. Controzzi, G. Lundborg, B. Rosen, M. C. Carrozza, and F. Sebelius, "Online myoelectric control of a dexterous hand prosthesis by transradial amputees," Ieee Transactions on Neural Systems and Rehabilitation Engineering, vol. 19, no. 3, pp. 260-270, 2011.

[30] F. C. Sebelius, B. N. Rosen, and G. N. Lundborg, "Refined myoelectric control in below-elbow amputees using artificial neural networks and a data glove," J Hand Surg Am, vol. 30, no. 4, pp. 780-9, 2005.

[31] G. C. Matrone, C. Cipriani, E. L. Secco, G. Magenes, and M. C. Carrozza, "Principal components analysis based control of a multi-dof underactuated prosthetic hand," J Neuroeng Rehabil, vol. 7, p. 16, 2010

[32] A. Fougner, E. Scheme, A. D. Chan, K. Englehart, and O. Stavdahl, "A multi-modal approach for hand motion classification using surface emg and accelerometers," Conf Proc IEEE Eng Med Biol Soc, vol. 2011, pp. 4247-50, 2011.

[33] A. M. Simon, B. A. Lock, and K. A. Stubblefield, "Patient training for functional use of pattern recognition-controlled prostheses," J Prosthet Orthot, vol. 24, no. 2, pp. 56-64, 2012, simon, Ann M Lock, Blair A Stubblefield, Kathy A R01 HD058000-01/HD/NICHD NIH HHS/ J Prosthet Orthot. 2012 Apr;24(2):56-64.

[34] K. Englehart and B. Hudgins, "A robust, real-time control scheme for multifunction myoelectric control," Ieee Transactions on Biomedical Engineering, vol. 50, no. 7, pp. 848-854, 2003.

[35] M. A. Oskoei and H. S. Hu, "Myoelectric control systems-a survey," Biomed Signal Process Control, vol. 2, no. 4, pp. 275-294, 2007.
[36] M. Khezri and M. Jahed, "Real-time intelligent pattern recognition algorithm for surface emg signals," Biomed Eng Online, vol. 6, p. 45, 2007.

[37] Y. Fang, X. Y. Zhu, and H. H. Liu, "Development of a surface emg acquisition system with novel electrodes configuration and signal representation," in 6th International Conference, ICIRA 2013, pp. 405414.

[38] J. P. M. Mogk and P. J. Keir, "Crosstalk in surface electromyography of the proximal forearm during gripping tasks," Journal of Electromyography and Kinesiology, vol. 13, no. 1, pp. 63-71, 2003.

[39] A. D. Chan and K. B. Englehart, "Continuous myoelectric control for powered prostheses using hidden markov models," IEEE Trans Biomed Eng, vol. 52, no. 1, pp. 121-4, 2005 